\title{
TFinDit: transcription factor-DNA interaction data depository
}

\author{
Daniel Turner, RyangGuk Kim and Jun-tao Guo*
}

\begin{abstract}
Background: One of the crucial steps in regulation of gene expression is the binding of transcription factor(s) to specific DNA sequences. Knowledge of the binding affinity and specificity at a structural level between transcription factors and their target sites has important implications in our understanding of the mechanism of gene regulation. Due to their unique functions and binding specificity, there is a need for a transcription factor-specific, structurebased database and corresponding web service to facilitate structural bioinformatics studies of transcription factorDNA interactions, such as development of knowledge-based interaction potential, transcription factor-DNA docking, binding induced conformational changes, and the thermodynamics of protein-DNA interactions.

Description: TFinDit is a relational database and a web search tool for studying transcription factor-DNA interactions. The database contains annotated transcription factor-DNA complex structures and related data, such as unbound protein structures, thermodynamic data, and binding sequences for the corresponding transcription factors in the complex structures. TFinDit also provides a user-friendly interface and allows users to either query individual entries or generate datasets through culling the database based on one or more search criteria.

Conclusions: TFinDit is a specialized structural database with annotated transcription factor-DNA complex structures and other preprocessed data. We believe that this database/web service can facilitate the development and testing of TF-DNA interaction potentials and TF-DNA docking algorithms, and the study of protein-DNA recognition mechanisms.
\end{abstract}

Keywords: Transcription factor, Database, Binding site prediction, Interaction potential

\section{Background}

Transcription factors (TFs) represent a distinct group of DNA binding proteins. They are sequence-specific while allowing certain degrees of variations at particular sites [1]. Though regulation of gene expression is a complicated biological process, one key step of this process is the binding of TFs to their DNA binding sites. At the genome level, identification of DNA target sites of transcription factors has been considered one of the grand challenges in post-genomic bioinformatics. The complex structures in Protein Data Bank (PDB) provide fine details about macromolecular interactions [2]. Knowledge of TF-DNA interactions can help us better understand the mechanisms of protein-DNA recognition, and more importantly, guide the design of new therapeutics

\footnotetext{
*Correspondence: jguo4@uncc.edu

Department of Bioinformatics and Genomics, College of Computing and Informatics, University of North Carolina at Charlotte, Charlotte, NC 28223, USA
}

for diseases in which transcription factors play critical roles [3-5]. Even though the number of TF-DNA complex structures in PDB has increased steadily due to technical advance in solving complex structures, it still only represents a small percentage of all the annotated transcription factors and their target DNA sites. At the same time, computational studies have made notable progress in modeling protein-DNA interactions. These include development of knowledge-based protein-DNA interaction potentials [6-8], investigation of binding affinity and specificity $[9,10]$, and protein-DNA docking studies [11-13]. Recently, structure-based TF binding site prediction has received much deserved attention owing to its ability to consider the position interdependence of TFs and the contribution of flanking sequences to binding specificity. The development of more accurate interaction potentials makes these structure-based methods feasible and more appealing in computational prediction of TF binding sites $[8,11,14]$. 
The paramount importance of transcription factors in gene regulation has attracted significant interests and efforts in developing TF resources either for one specific genome, such as RegulonDB for E. coli K-12 [15] and EDGEdb for C. elegans [16], or for one specific kingdom, such as JAPAR for Eukaryotes [17] and RegTransBase for bacteria [18]. The TF resources currently available across the tree of life are listed in a recent survey [19]. Most of these TF resources have either manually annotated or computationally predicted TFs while others use a combination of both annotation approaches. Though these TF resources contain large amounts of data that are valuable to study the diversity and evolution of transcription factors, they are not designed for structural bioinformatics studies of TF-DNA interactions.

On the other hand, several databases/web servers about general protein-nucleic acids interactions have been developed. These include AANT [20], ProNIT [21], NPIDB [22], PDA [23], BIPA [24], hPDI [25], 3Dfootprint [26], PDIdb [27], ccPDB [28] and others. While each database/web server offers search options on certain aspects about general protein-nucleic acid interactions, the unique characteristics of transcription factors and the imperative goal of structure-based TF-binding site prediction call for a TF-specific database/web server, especially when transcription factors are not well classified and annotated in PDB. In addition, previous studies have revealed different interaction "modes" between transcription factors and other types of DNA binding proteins [29,30]. To the best of our knowledge, there are no TF-specific structural databases/web services available.

We developed TFinDit (for Transcription Factor-DNA interaction Data depository) to facilitate structural bioinformatics studies of TF-DNA interactions. TFinDit offers annotated TF-DNA complex structures and other useful information, such as unbound TF structures, thermodynamic data of TF-DNA complexes, and automatic mapping between TF-DNA complexes and known TF binding sites. TFinDit also provides a web interface with multiple search options. Potential users can generate datasets based on their research needs in studying TF-DNA interaction, such as bound-unbound TF pairs, DNA binding sites, and thermodynamic data for wildtype and/or mutants (TF and DNA), or focus on the structural details of one specific TF-DNA complex. The framework of TFinDit can be easily extended to include more useful information once identified in the future.

\section{Construction and content}

Computationally, TFinDit has two major components: a relational database using MySQL 5.0.45 and a web server providing an interface accessible to potential users to search the database and display the search results. The web server is developed with a combination of PHP 5.1.6, Java JDK v1.6.0, Python 2.4.3, and Apache Web Server 2.3.3.

The database contains all TF-DNA complexes from PDB [2]. The collection of TF-DNA complexes from $\mathrm{PDB}$ is not trivial since the classification of some DNAbinding proteins in PDB is ambiguous. For example, transcription factors Escherichia coli SigmaE Region 4, $2 \mathrm{H} 27$ [31] and the ribbon-helix-helix domain of Escherichia coli PutA, 2RBF [32] are classified as "transferase" and "oxidoreductase" respectively in PDB. So we first developed an in-house program that can automatically identify transcription factors in PDB by combining information from Gene Ontology (GO) terms [33], PDB keywords, and UniProt keywords [34]. The procedure of the annotation process is shown in Additional file 1 Figure S1. The script and related files are available for download from the TFinDit site (Resources Tab).

The procedure for generating the initial data and for future updates is shown in Figure 1. Briefly, all the DNA-binding proteins are culled from PDB. The TFDNA complexes with double-strand DNA are selected using our in-house TF-annotation program that takes PDB IDs as inputs. The list of TF-DNA complexes will serve as the base for getting other data and for preprocessing. The first step in preprocessing is to search for homologous TF-DNA complexes and homologous TF structures in free state (unbound structures) with at least $80 \%$ sequence identity to the query bound TF structures. Data from both the sequential (similarity, coverage, etc.) and structural comparisons are stored in the database (Figure 1). TF structural comparison is carried out with TM-align that uses TM-score for alignment optimization [35-37]. The TM-score is normalized independent on the protein's size and is more sensitive to global structure changes than to local structure changes compared to RMSD (Root Mean Square Deviation) [35]. While RMSD is a widely used metric for structural differences, TM-score is more suitable for spotting global structure changes [35-37]. In addition, previous studies have shown that the activation regions of transcription factors have higher degree of disorder and transcription factors in eukaryote have more disordered regions than those in prokaryote [38-40]. Neither TM-score nor RMSD could reflect the structural differences caused by missing residues or disordered regions in TF structures. After structural alignment, both the TM-scores and the RMSD values are calculated using the $\mathrm{C}$-alpha of the amino acids between the unbound and bound TF structures and are stored in the database. Currently, the database contains 1391 bound and 2370 unbound chains.

Another important component in preprocessing is the mapping of TF structures to entries in other important databases. These include databases with TF binding sites 


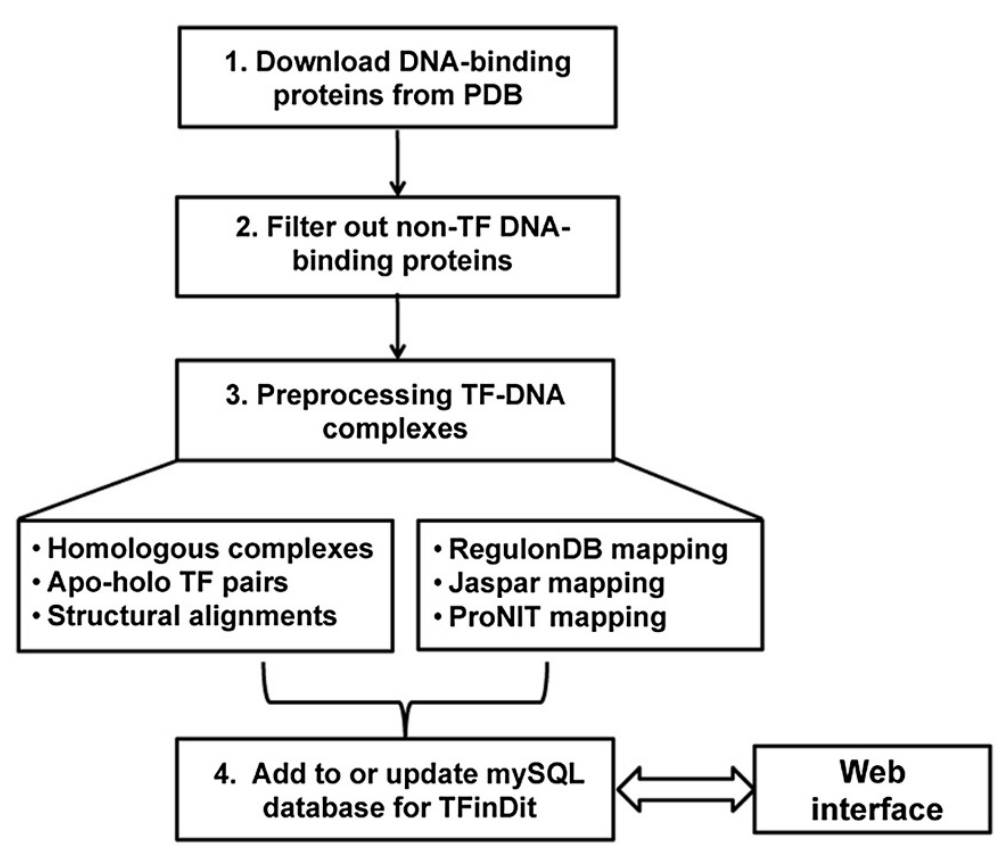

Figure 1 Procedure for TFinDit construction and update.

(RegulonDB and Jaspar) [15,17] and ProNIT, a thermodynamic database for protein-nucleic interactions [21]. Among the 1391 bound TF chains in current release, 307 have ProNIT entries and 433 have annotated binding sequences from RegulonDB/Jaspar. After the preprocessing step, all the data are stored in a relational database. The same procedure will be used for future updates and newly identified entries and related data will be added to the database (Figure 1). We plan to update the database every two to three months.

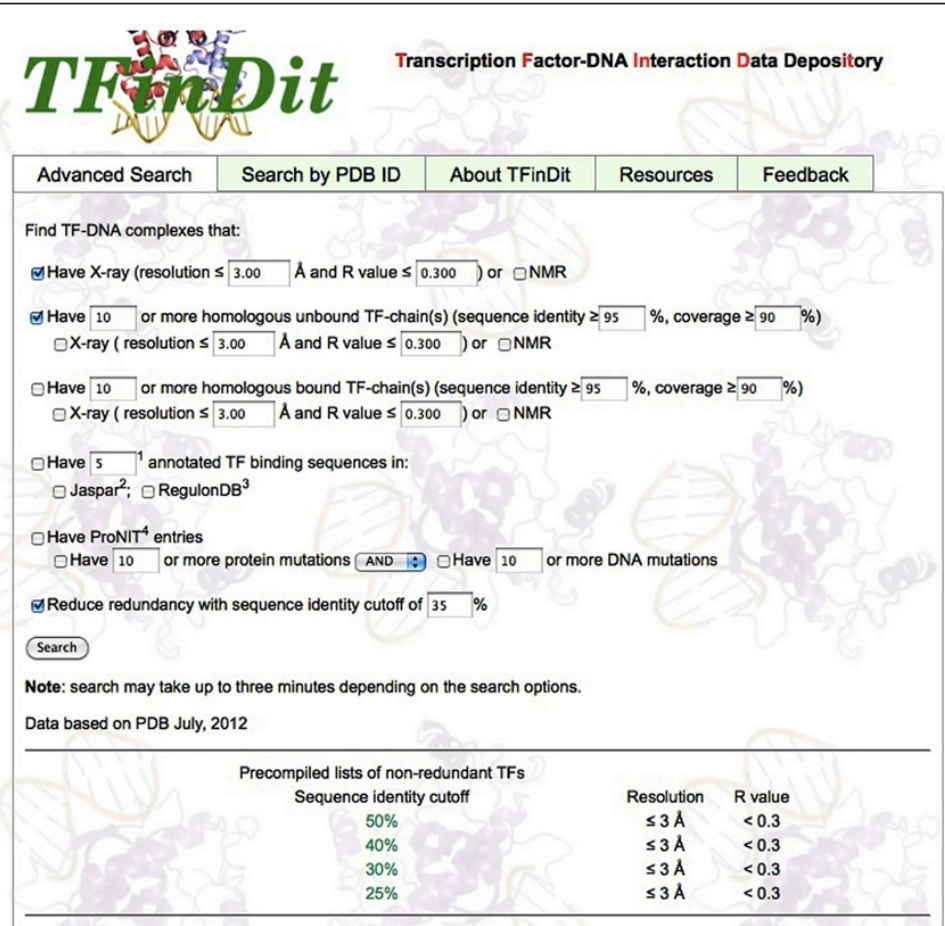

Figure 2 A snapshot of the "Advanced Search" page for TFinDit. 


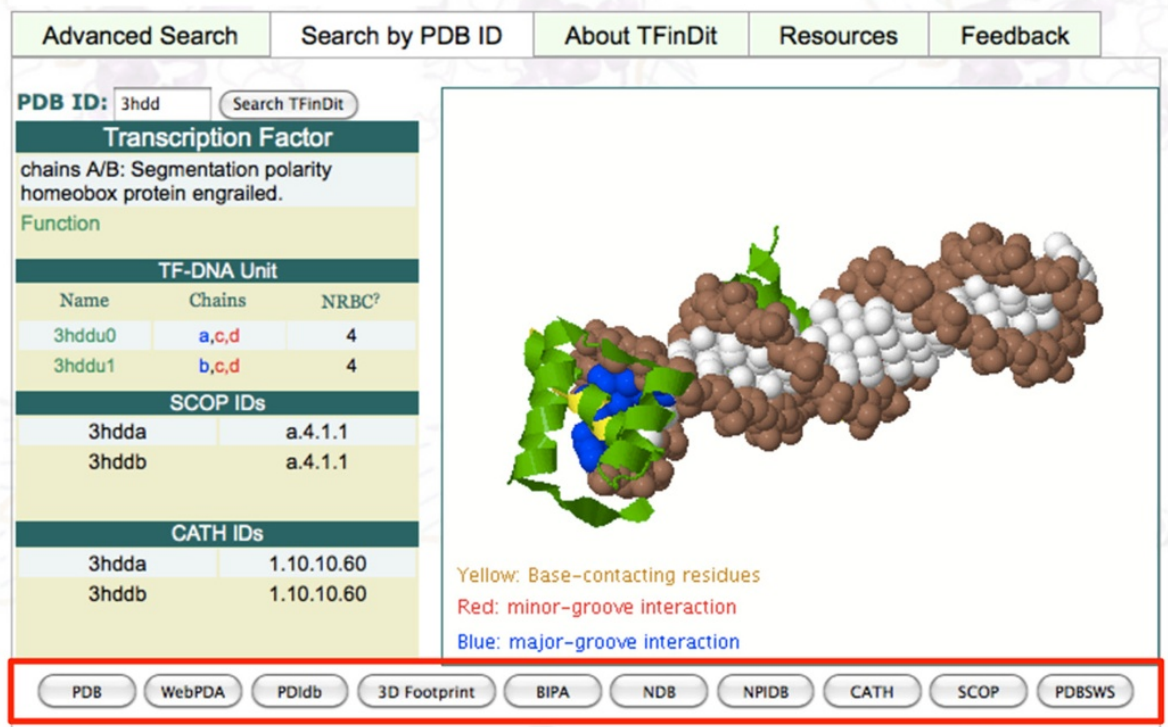

\begin{tabular}{|lccccccc|}
\hline \multicolumn{7}{|c|}{ Homologous Unbound TF-Chain(s) } \\
\hline $\begin{array}{l}\text { Bound } \\
\text { Chain }\end{array}$ & $\begin{array}{c}\text { Unbound } \\
\text { Chain }\end{array}$ & $\begin{array}{c}\text { Sequence } \\
\text { Identity }\end{array}$ & E-Value & $\begin{array}{c}\text { Hit? } \\
\text { Coverage }\end{array}$ & $\begin{array}{c}\text { Query? } \\
\text { Coverage }\end{array}$ & TMScore & RMSD? $^{\text {Therla }}$ \\
\hline 3hddb & 2jwta & 100 & $1.0 \mathrm{e}-26$ & 96.7 & 98.3 & 0.895 & 1.29 \\
3hddb & 1p7jd & 98.3 & $4.0 \mathrm{e}-26$ & 100 & 98.3 & 0.913 & 0.66
\end{tabular}

\begin{tabular}{|c|c|c|c|c|c|c|c|}
\hline \multicolumn{8}{|c|}{ Homologous Bound TF-Chain(s) } \\
\hline $\begin{array}{l}\text { Query } \\
\text { Chain }\end{array}$ & $\begin{array}{l}\text { Homologous } \\
\text { Chain }\end{array}$ & $\begin{array}{l}\text { Sequence } \\
\text { Identity }\end{array}$ & E-Value & $\begin{array}{c}\text { Hit }^{?} \\
\text { Coverage }\end{array}$ & $\begin{array}{c}\text { Query? } \\
\text { Coverage }\end{array}$ & TMScore & RMSD? \\
\hline 3hddb & 3hdda & 100 & $6.00-30$ & 100 & 100 & 0.938 & 0.42 \\
\hline 3hddb & 1hddd & 100 & $2.0 \mathrm{e}-29$ & 96.7 & 98.3 & 0.981 & 0.34 \\
\hline 3hddb & 1hddc & 100 & $2.0 \mathrm{e}-29$ & 96.7 & 98.3 & 0.933 & 1.22 \\
\hline 3hddb & $2 h d d b$ & 98.3 & $7.0 \mathrm{e}-29$ & 96.7 & 98.3 & 0.964 & 0.35 \\
\hline
\end{tabular}

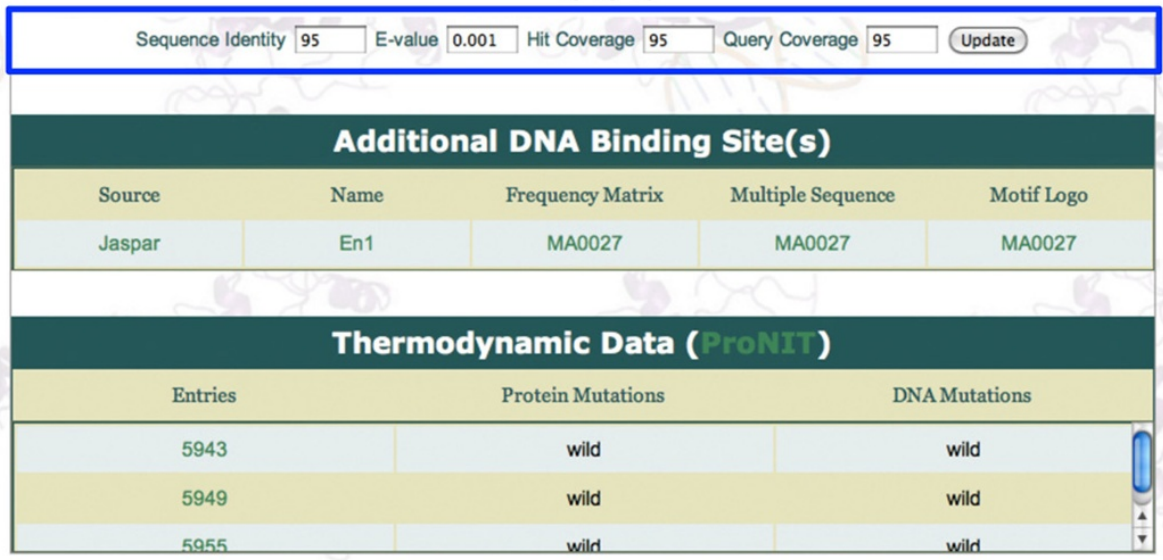

Figure 3 Detailed information for TFinDit entry 3HDD. The red box indicates the quick links to other analysis tools. The blue box shows the cutoff values that users can change and get updated data. 


\section{Utility and discussion}

The web interface offers two options for queries. One is for culling non-redundant datasets for different research purposes. For example, users can generate a nonredundant dataset of bound-unbound pairs for studying conformational changes after TF-DNA binding or docking studies. Other useful datasets that can be generated include homologous TF-DNA complexes, TF-DNA complexes with thermodynamic data for both wild-type and/ or mutant molecules, and TF-DNA complexes with experimentally validated binding sequences (Figure 2). Users can specify the resolution for x-ray structures, the sequence identity and coverage for homologous sequences, and the minimum number of entries that satisfy the selection criteria. PISCES is used to remove redundancy [41].

The other search option allows the retrieval of detailed structural and related data for a specific TF-DNA complex in TFinDit. An example for PDB ID 3HDD [42] is shown in Figure 3. These data include the homologous unbound transcription factors, homologous TF-DNA complexes, known annotated additional binding sequences, and thermodynamic data for the wild-type and mutants of the complexes in ProNIT (Figure 3). The sequence identity, coverage, and the structural differences between homologous bound-unbound or bound-bound pairs in terms of both the TM-Score measure and RMSD, are also displayed. Users also have the option to change the cutoffs for sequence identity, $E$-value, coverage (Blue Box in Figure 3). In addition, links of the TFinDit entry to other useful web services are also provided (Red Box in Figure 3). These include PDB [2], WebPDA [23], PDIdb [27], 3D-footprint [26], BIPA [24], NDB [43], and NPIDB [22] and to structural classifications websites CATH [44] and SCOP [45]. Users can get a quick access to all the related predictive or analysis tools for each TF-DNA entry from TFinDit. On the "Resources" page, a number of useful predictive tools for modeling TF-DNA interactions and other services are provided and the list will be updated when more tools are identified. Current tools include TF-Modeller for building comparative TF-DNA complex models [46] and DDNA3 for DNA binding domain prediction [47], our in-house program for TF annotation, and some services listed in the quick-link box (Figure 3).

\section{Conclusions}

TFinDit is a specialized structural database with annotated transcription factor-DNA complex structures and other related data. We believe that this database/web service can facilitate structural bioinformatics studies, especially in the development of TF-DNA interaction potentials, the testing of TF-DNA docking algorithms, and the study of protein-DNA recognition mechanisms.

\section{Availability and requirements}

The service is available at http://bioinfozen.uncc.edu/ tfindit

\section{Additional file}

Additional file 1: Figure S1. Flowchart for identifying TF-DNA

complexes in PDB.

\section{Abbreviations}

PDB: Protein Data Bank; RMSD: Root Mean Square Deviation; TF: Transcription Factor.

\section{Competing interests}

The authors declare that they have no competing interests.

\section{Authors' contributions}

DT implemented the database and the web service. RK participated in the design and the initial implementation of TFinDit. JTG conceived the study, participated in the design, and wrote the manuscript. All authors read and approved the final manuscript.

\section{Acknowledgements}

We would like to thank Ms. Akshita Dutta and Ms. Rosario I. Corona for their help with the project. This work was supported by the National Science Foundation \#DBI0844749 to JTG.

Received: 18 April 2012 Accepted: 23 August 2012

Published: 3 September 2012

\section{References}

1. Pan Y, Tsai CJ, Ma B, Nussinov R: Mechanisms of transcription factor selectivity. Trends Genet 2010, 26:75-83.

2. Berman HM, Westbrook J, Feng Z, Gilliland G, Bhat TN, Weissig H, Shindyalov IN, Bourne PE: The Protein Data Bank. Nucleic Acids Res 2000, 28:235-242.

3. Kuntz ID: Structure-based strategies for drug design and discovery. Science 1992, 257:1078-1082.

4. Darnell JE Jr: Transcription factors as targets for cancer therapy. Nat Rev Cancer 2002, 2:740-749.

5. Sankpal UT, Goodison S, Abdelrahim M, Basha R: Targeting Sp1 transcription factors in prostate cancer therapy. Med Chem 2011, 7:518-525.

6. Liu Z, Mao F, Guo JT, Yan B, Wang P, Qu Y, Xu Y: Quantitative evaluation of protein-DNA interactions using an optimized knowledge-based potential. Nucleic Acids Res 2005, 33:546-558.

7. Robertson TA, Varani G: An all-atom, distance-dependent scoring function for the prediction of protein-DNA interactions from structure. Proteins 2007, 66:359-374.

8. Xu B, Yang Y, Liang H, Zhou Y: An all-atom knowledge-based energy function for protein-DNA threading, docking decoy discrimination, and prediction of transcription-factor binding profiles. Proteins 2009, 76:718-730.

9. Ashworth J, Baker D: Assessment of the optimization of affinity and specificity at protein-DNA interfaces. Nucleic Acids Res 2009, 37:e73.

10. Luscombe NM, Thornton JM: Protein-DNA interactions: amino acid conservation and the effects of mutations on binding specificity. $J \mathrm{Mol}$ Biol 2002, 320:991-1009.

11. Liu Z, Guo JT, Li T, Xu Y: Structure-based prediction of transcription factor binding sites using a protein-DNA docking approach. Proteins 2008, 72:1114-1124.

12. van Dijk M, Bonvin AM: Pushing the limits of what is achievable in protein-DNA docking: benchmarking HADDOCK's performance. Nucleic Acids Res 2010, 38:5634-5647.

13. van Dijk M, van Dijk AD, Hsu V, Boelens $R$, Bonvin AM: Information-driven protein-DNA docking using HADDOCK: it is a matter of flexibility. Nucleic Acids Res 2006, 34:3317-3325.

14. Angarica VE, Perez AG, Vasconcelos AT, Collado-Vides J, Contreras-Moreira B: Prediction of TF target sites based on atomistic models of protein-DNA complexes. BMC Bioinforma 2008, 9:436. 
15. Gama-Castro S, Salgado H, Peralta-Gil M, Santos-Zavaleta A, Muniz-Rascado L, Solano-Lira H, Jimenez-Jacinto V, Weiss V, Garcia-Sotelo JS, Lopez-Fuentes $A$, et al: RegulonDB version 7.0: transcriptional regulation of Escherichia coli K-12 integrated within genetic sensory response units (Gensor Units). Nucleic Acids Res 2011, 39:D98-D105.

16. Barrasa MI, Vaglio P, Cavasino F, Jacotot L, Walhout AJ: EDGEdb: a transcription factor-DNA interaction database for the analysis of $C$. elegans differential gene expression. BMC Genomics 2007, 8:21.

17. Portales-Casamar E, Thongjuea S, Kwon AT, Arenillas D, Zhao X, Valen E, Yusuf D, Lenhard B, Wasserman WW, Sandelin A: JASPAR 2010: the greatly expanded open-access database of transcription factor binding profiles. Nucleic Acids Res 2010, 38:D105-D110.

18. Kazakov AE, Cipriano MJ, Novichkov PS, Minovitsky S, Vinogradov DV, Arkin A, Mironov AA, Gelfand MS, Dubchak I: RegTransBase-a database of regulatory sequences and interactions in a wide range of prokaryotic genomes. Nucleic Acids Res 2007, 35:D407-D412.

19. Charoensawan V, Wilson D, Teichmann SA: Genomic repertoires of DNAbinding transcription factors across the tree of life. Nucleic Acids Res 2010, 38:7364-7377.

20. Hoffman MM, Khrapov MA, Cox JC, Yao J, Tong L, Ellington AD: AANT: the Amino Acid-Nucleotide Interaction Database. Nucleic Acids Res 2004, 32:D174-D181.

21. Kumar MD, Bava KA, Gromiha MM, Prabakaran P, Kitajima K, Uedaira H, Sarai A: ProTherm and ProNIT: thermodynamic databases for proteins and protein-nucleic acid interactions. Nucleic Acids Res 2006, 34:D204-D206.

22. Spirin S, Titov M, Karyagina A, Alexeevski A: NPIDB: a database of nucleic acids-protein interactions. Bioinformatics 2007, 23:3247-3248.

23. Kim R, Guo JT: PDA: an automatic and comprehensive analysis program for protein-DNA complex structures. BMC Genomics 2009, 10(Suppl 1):S13.

24. Lee $S$, Blundell TL: BIPA: a database for protein-nucleic acid interaction in 3D structures. Bioinformatics 2009, 25:1559-1560.

25. Xie Z, Hu S, Blackshaw S, Zhu H, Qian J: hPDI: a database of experimental human protein-DNA interactions. Bioinformatics 2010, 26:287-289.

26. Contreras-Moreira B: 3D-footprint: a database for the structural analysis of protein-DNA complexes. Nucleic Acids Res 2010, 38:D91-D97.

27. Norambuena T, Melo F: The Protein-DNA Interface database. BMC Bioinforma 2010, 11:262.

28. Singh H, Chauhan JS, Gromiha MM, Raghava GP: ccPDB: compilation and creation of data sets from Protein Data Bank. Nucleic Acids Res 2012, 40: D486-D489.

29. Contreras-Moreira B, Sancho J, Angarica VE: Comparison of DNA binding across protein superfamilies. Proteins 2010, 78:52-62.

30. Kim R, Corona Rl, Hong B, Guo JT: Benchmarks for flexible and rigid transcription factor-DNA docking. BMC Struct Biol 2011, 11:45.

31. Lane WJ, Darst SA: The structural basis for promoter -35 element recognition by the group IV sigma factors. PLOS Biol 2006, 4:e269.

32. Zhou Y, Larson JD, Bottoms CA, Arturo EC, Henzl MT, Jenkins JL, Nix JC, Becker DF, Tanner JJ: Structural basis of the transcriptional regulation of the proline utilization regulon by multifunctional PutA. J Mol Biol 2008, 381:174-188.

33. Ashburner M, Ball CA, Blake JA, Botstein D, Butler H, Cherry JM, Davis AP, Dolinski K, Dwight SS, Eppig JT, et al: Gene ontology: tool for the unification of biology. The Gene Ontology Consortium. Nat Genet 2000, 25:25-29.

34. Wu CH, Apweiler R, Bairoch A, Natale DA, Barker WC, Boeckmann B, Ferro S, Gasteiger E, Huang H, Lopez R, et al: The Universal Protein Resource (UniProt): an expanding universe of protein information. Nucleic Acids Res 2006, 34:D187-D191.

35. Zhang Y, Skolnick J: Scoring function for automated assessment of protein structure template quality. Proteins 2004, 57:702-710.

36. Zhang Y, Skolnick J: TM-align: a protein structure alignment algorithm based on the TM-score. Nucleic Acids Res 2005, 33:2302-2309.

37. $\mathrm{Xu} \mathrm{J}$, Zhang $\mathrm{Y}$ : How significant is a protein structure similarity with TMscore $=0.5$ ? Bioinformatics 2010, 26:889-895.

38. Liu J, Perumal NB, Oldfield CJ, Su EW, Uversky VN, Dunker AK: Intrinsic disorder in transcription factors. Biochemistry 2006, 45:6873-6888.

39. Minezaki Y, Homma K, Kinjo AR, Nishikawa K: Human transcription factors contain a high fraction of intrinsically disordered regions essential for transcriptional regulation. J Mol Biol 2006, 359:1137-1149.

40. Dunker AK, Uversky VN: Drugs for 'protein clouds': targeting intrinsically disordered transcription factors. Curr Opin Pharmacol 2010, 10:782-788.
41. Wang G, Dunbrack RL Jr: PISCES: a protein sequence culling server. Bioinformatics 2003, 19:1589-1591.

42. Fraenkel E, Rould MA, Chambers KA, Pabo CO: Engrailed homeodomainDNA complex at 2.2 A resolution: a detailed view of the interface and comparison with other engrailed structures. J Mol Biol 1998, 284:351-361.

43. Berman HM, Westbrook J, Feng Z, lype L, Schneider B, Zardecki C: The Nucleic Acid Database. Acta Crystallogr D: Biol Crystallogr 2002, 58:889-898.

44. Orengo CA, Michie AD, Jones S, Jones DT, Swindells MB, Thornton JM: CATH-a hierarchic classification of protein domain structures. Structure 1997, 5:1093-1108.

45. Murzin AG, Brenner SE, Hubbard T, Chothia C: SCOP: a structural classification of proteins database for the investigation of sequences and structures. J Mol Biol 1995, 247:536-540.

46. Contreras-Moreira B, Branger PA, Collado-Vides J: TFmodeller: comparative modelling of protein-DNA complexes. Bioinformatics 2007, 23:1694-1696.

47. Zhao H, Yang Y, Zhou Y: Structure-based prediction of DNA-binding proteins by structural alignment and a volume-fraction corrected DFIREbased energy function. Bioinformatics 2010, 26:1857-1863.

doi:10.1186/1471-2105-13-220

Cite this article as: Turner et al.: TFinDit: transcription factor-DNA

interaction data depository. BMC Bioinformatics 2012 13:220.

\section{Submit your next manuscript to BioMed Central and take full advantage of:}

- Convenient online submission

- Thorough peer review

- No space constraints or color figure charges

- Immediate publication on acceptance

- Inclusion in PubMed, CAS, Scopus and Google Scholar

- Research which is freely available for redistribution

Submit your manuscript at www.biomedcentral.com/submit
C) Biomed Central 